\title{
Systems for Safety and
}

Autonomous Behavior in Cars: The DARPA Grand Challenge Experience

\section{Car safety systems are helping drivers to stay in their lanes, improving their night vision and are making driving decisions where millisecond response is needed.}

By Ümit Özgüner, Member IEEe, Christoph Stiller, Senior Member IEEE, And

Keith Redmill, Member IEEE

ABSTRACT | In this paper, we review technologies for autonomous ground vehicles and their present capabilities in research and in the automotive market. We outline technology requirements for enhanced functions and for infrastructure development. Since the recent Grand Challenge competition is a major force to advance technology in this field, we specifically refer to our experiences in developing a participating vehicle. We present a multisensor platform that has been proven in an off-road environment. It combines different sensing modalities that inherently yield uncertain information. Finite-state machines are formulated to generate rule-based autonomous behavior that enables fully autonomous off-road driving. Overall, the intent of the paper is to evaluate approaches and technologies used in the two Grand Challenges as they contribute to the needs of autonomous cars on the road.

KEYWORDS | Autonomous systems; autonomous vehicles; cars; intelligent vehicles; safety; sensor fusion

\section{INTRODUCTION}

\section{A. Background in Autonomy in Cars}

One of the most fascinating trends in automotive technology is the introduction of capabilities to provide

Manuscript received December 28, 2005; revised July 3, 2006

ü. Özgüner and $\mathbf{K}$. Redmill are with the Department of Electrical and

Computer Engineering, The Ohio State University, Columbus, OH 43210 USA

(e-mail: umit@ece.osu.edu; redmill@ece.osu.edu).

c. Stiller is with Institute für Mess-und Relegungstechnik, University of Karlsruhe, 76131 Karlsruhe, Germany (e-mail: stiller@mrt.uka.de).

Digital Object Identifier: 10.1109/JPROC.2007.888394 autonomous behavior to cars. It is expected that this will not only show quantitative effects on traffic, but in the long term will provide a new quality of traffic operation including concerted navigation for safe, comfortable, and efficient driving.

We define "autonomy" in a car as the car making driving decisions without intervention of a human. As such, autonomy exists in various aspects of a car today: "cruise control" and "Antilock Brake Systems" (ABS) are prime examples of systems exhibiting autonomous behavior. Additional systems already exist in a few models, including advanced cruise control, lane keeping support, lane change warning, and obstacle avoidance systems, all of which expand the range of autonomous behavior. Near-term developments that we anticipate, some first appearing as warning devices, include intersection collision warning, backup parking, parallel parking aids, and bus precision docking, show either autonomous behavior or can be totally autonomous with the addition of actuation. Finally, truck convoys and driverless busses in enclosed areas have seen limited operational deployment.

Studies in autonomous behavior for cars, concentrating on sensing, perception and control, have been ongoing for a number of years. One can list a number of capabilities, beyond basic speed regulation, that are key to autonomous behavior. These will all affect the cars of the future.

- Car following/convoying.

- Lane keeping/lane change.

- Emergency stopping.

- Collision mitigation/obstacle avoidance.

- $\quad$ Routing and traffic control capabilities. 
In each of the above operations, the car is expected to do self-sensing (basically speed and acceleration), sensing with respect to some absolute coordinate system (usually using global positioning system (GPS) augmented with inertial or dead reckoning sensors and possibly the help of a map data base) and sensing with respect to the immediate environment (with respect to lane markings, special indicators on the road, obstacles, other vehicles, traffic signs, and signals, etc.) We shall be discussing some of the relevant technologies.

Large public demonstrations, challenges, and races serve two purposes. The first is to provide an impetus for rapid advances in the development and integration of research and related technologies; the second is to focus public attention and ultimately lead to acceptance of and support for such technologies.

The DARPA Grand Challenges of 2004 and 2005 certainly served the second purpose, in providing a stage for media attention, illustrating the technologies and even providing a certain amount of color and drama.

In this paper, we shall concentrate on the first aspect by attempting to review where the Grand Challenges fell in the general development of autonomous vehicle technologies. We shall review briefly the state-of-the-art and trends in related vehicle technologies. Our exposure will be inevitably weighed towards our own vehicle in GC'05. Although our platform was not a standard car, the technologies and approaches used were fairly representative.

The remainder of this paper is organized as follows. After recalling several public demonstrations of the status of research, we provide an overview of autonomous systems that have already entered the automotive market. Section II outlines technology requirements for enhanced functions and for infrastructure development. The Grand Challenge competition outlined in Section III has been an impetus to advance technology in this field. Sections IV and V will outline sensing and control strategies of our Grand Challenge vehicle ION, respectively, while Section VI concludes with lessons learned and implications for future automobiles.

\section{B. Background in Autonomous Driving Demonstrations}

Through the years, many demonstrations have been held showing the capabilities of autonomous vehicles, and by extension, the underlining sensor capabilities. One of the most comprehensive highway-based demonstrations was held in 1997 on I-15 in San Diego, CA, and showed the capabilities of cars, busses, and trucks in various automated highway scenarios. This demonstration (called Demo'97) was organized by the National Automated Highway Systems Consortium (see, e.g., [34], [35], and [41]). The key technologies were lane-following using roadway embedded magnets, roadway laid radar-reflective stripes, or existing visible lane markers detected with vehicle mounted cameras; car following using laser or

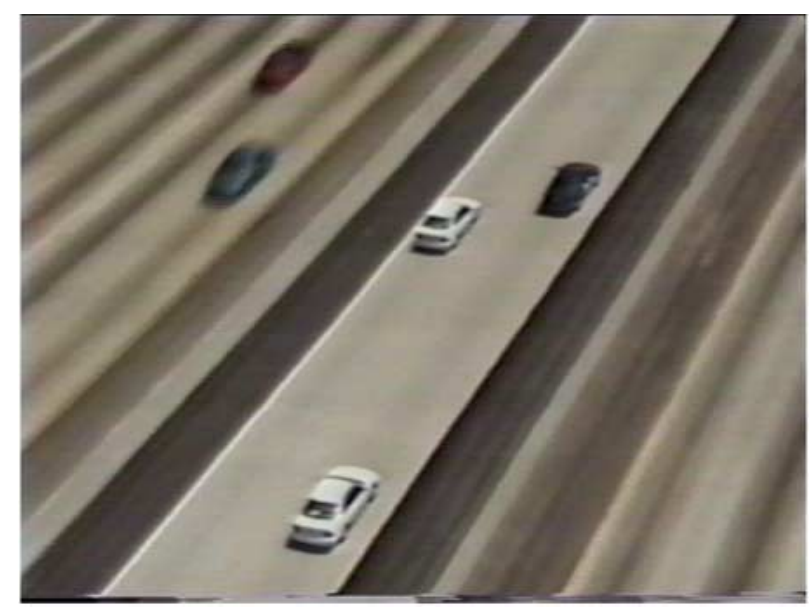

Fig. 1. Two autonomous cars from The Ohio State University team in Demo'97 performing a vehicle pass without infrastructure aids.

radar, with or without the help of intervehicle communication; and the management of multiple vehicles to carry out a specific scenario. Scenario maintenance was accomplished either by an entirely timed and scripted program, by GPS and location-based triggering, or by situation-based triggering (e.g. Fig. 1).

Demo'97 was followed by a number of demonstrations around the world, notably in Japan, Holland, France, and the U.S. In each case, not only were state-of-the-art technologies demonstrated, but the public was informed about what to expect in future cars (see, e.g., [1], [4], and [17]).

The DARPA Grand Challenge, which was held in March 2004 and October 2005, stressed the nonstructured environment of off-road desert driving. Although some aspects of the technical problems addressed were different, the technology set utilized was very similar to that used for autonomous highway or urban driving. It was also another step in raising public awareness and expectations for future cars.

\section{Development in the Automotive Market}

We are currently witnessing driver assistance systems with environmental sensing capabilities entering the automotive market. For many reasons, including market acceptance and liability, those innovations are frequently first introduced into Japanese models before they appear in upper class models of the European and American automotive industry. The following nonexhaustive overview shows the ongoing search for the most suitable sensor technology and appropriate functions that can be introduced despite today's technical limitations.

Starting with the Mitsubishi Diamante in 1995 and followed by Toyota in 1996, Adaptive Cruise Control (ACC) Systems employed automotive lidar or radar sensors to measure the distance, velocity, and heading angle of preceding vehicles. This information is used to 
improve on the longitudinal control of conventional Cruise Control systems. When a free roadway is detected the system behaves like a conventional Cruise Control. When a slower preceding vehicle is detected, the ACC system follows at a safe driving distance until the situation changes, e.g., due to a lane change maneuver of either vehicle. The system works well on highways or in similar operation conditions. It is designed as a comfort enhancing system, i.e., it neither primarily aims to, nor possesses sufficient sensor performance to provide safe longitudinal control by itself. The responsibility is kept with the driver, and hence the operational domain is restricted to highway speeds and to a comfortable maximum deceleration, for example $2,5 \mathrm{~m} / \mathrm{s}^{2}$. In European cars, the Mercedes S-Class introduced ACC (called Distronic) in 1999 followed by Jaguar's XKR the same year and BMW's 7-series in early 2000. Despite initial and some remaining deficiencies, which mainly stem from the sensors, ACC systems have since become widely accepted in upper class vehicles. Function enhancements that allow seamless operation from highway cruising to stop-and-go traffic are under development [45].

Video sensors were first introduced for lane departure warning (LDW) systems. These systems observe the white lane markings on the road and warn the driver when an unintended lane departure is detected. Market introduction has first focused on trucks such as the Mercedes or MAN flagships. In America, the market was first approached by the small company AssistWare (http://www. assistware.com) that was later acquired by Cognex.

Night vision systems assist the driver in bad visibility conditions such as night driving by displaying an image as observed by a sensor that can cope with such conditions. GM first introduced night vision in its Lincoln Navigator employing a far infrared (FIR) spectrum camera. The BMW 7-series followed in 2005 almost immediately with an alternative near infrared (NIR) concept and the Mercedes S-Class employed a CMOS camera and NIR high beam headlamps.

In 2002, Honda introduced a lane keeping assist system called the Honda intelligent driver support system (HIDS) to the Japanese market [20], [39]. It combines ACC with lane keeping support. Based on lane detection by a video sensor, an auxiliary supportive momentum is added to the driver's action. With the collision mitigation system (CMS), Honda has followed an escalation strategy to mitigate collisions. Beginning with activation of a warning signal when the headway of the vehicle becomes small CMS enhances its intervention to moderate braking deceleration that subsequently increases to about $6 \mathrm{~m} / \mathrm{s}^{2}$ as the danger of a collision increases. Should the driver react in this situation, but his reaction is insufficient to avoid the accident, the system enhances his action during all phases. Even though the system cannot avoid accidents completely, the support of the driver and the speed reduction will reduce collision severity [24].
Since 2005, a radar-based active brake assistance system that supports the driver to avoid or mitigate frontal collisions by autonomous braking is also available in the Mercedes S-class.

Blind spot information systems have been introduced in 2006 into several Volvo cars based on video cameras and in Audi based on short-range radar.

\section{SOME TECHNOLOGY NEEDS IN FUTURE CARS}

\section{A. Functional Needs}

Despite the tremendous achievements of research in driver assistance systems over the past decades, the automatic control capabilities of vehicles are still far behind the capabilities of human drivers. The previous section has listed some driver assistance functions that have been introduced to the market. However, the current contribution of driver assistance systems to road safety falls behind many optimistic expectations. A major cause for the slower than expected pace is that driver assistance systems require interdisciplinary work in many different fields including sensor technology, machine perception, control engineering, artificial intelligence, driver psychology, human machine interaction, market acceptance, and legal issues and liability. While the long-term perspective of safe driving is convincing from any point of view, low hanging fruits are difficult to find. The identification of intermediate functions that can cope with the deficiencies of today's perception capabilities and produce transparent and predictable system behavior that improves driving safety is an ongoing challenge. The few advantages of current automated cognition over human cognition include the capability of immediate action within a few milliseconds and the indefatigable awareness of machines and nighttime perception. It is interesting to note that today's driver assistance systems are arranged just in these niches.

Fig. 2 depicts a potential evolution of functions together with their sensor requirements. These emerge from existing functions for vehicle dynamic stabilization that require sensing of vehicle internal quantities, for example, the velocity of the individual wheels, acceleration, or yaw rate. These signals allow functions that stabilize the vehicle in extreme driving situations, such ABS, electronic stability program (ESP), or anti-skid control (ASC). ${ }^{1}$

The availability of environment sensing by sonar, radar, lidar, or video opens up new information and comfort functions, but due to the associated sensor uncertainty, the final responsibility has to stay with the driver. Prominent examples for such functions are parking assistance systems that signal the driver the distance to rearwards obstacles or display an image of a rear view camera, while leaving

${ }^{1}$ While ABS and ASC control individual wheel slip under braking and acceleration, respectively, ESP controls yaw rate of the vehicle. 
vehicle control with the driver. Longitudinal control assistance systems require information on obstacles in the vehicles path. The limited braking capabilities of ACC are a tribute to the remaining uncertainty of lidar and radar sensors. Likewise, lateral control systems require information on the road geometry ahead and the vehicles' pose relative to the road. This information may stem from video sensors or may be acquired with the help of a dedicated infrastructure (see Section II-B). Again, fully automated steering necessitates sensor reliability under all circumstances.

It may not be possible to resolve the uncertainty of a single sensor to a sufficient degree for safety functions, such as emergency braking or collision avoidance, in the near future. Hence, multisensor systems are under development employing information fusion strategies to exploit the diversity of different sensor technologies. Information fusion techniques (see e.g., [25] for an overview) not only combine the fields of view of different sensors but may also allow the system to maintain functionality in case of a failure or a missed detection of any single sensor [37]. While multiple sensor systems provide physical diversity at a particularly strong level, cost considerations restrict the implementation of a multitude of sensor systems in many practical applications.

As the equipment rate of vehicles with capabilities for environmental sensing increases, it becomes likely that a vehicle possesses information about the environment that is relevant to others. This gives rise to the introduction of vehicle-to-vehicle or vehicle-to-infrastructure communication into future cars [2], [26]. Rearward traffic may, thus, enhance its "range of view" via telematics, enabling timely reaction to congestions and other traffic irregularities [30]. A motivation for a rather local exchange of sensor information is illustrated by the specific scenario of a street crossing shown in Fig. 3. The environment information

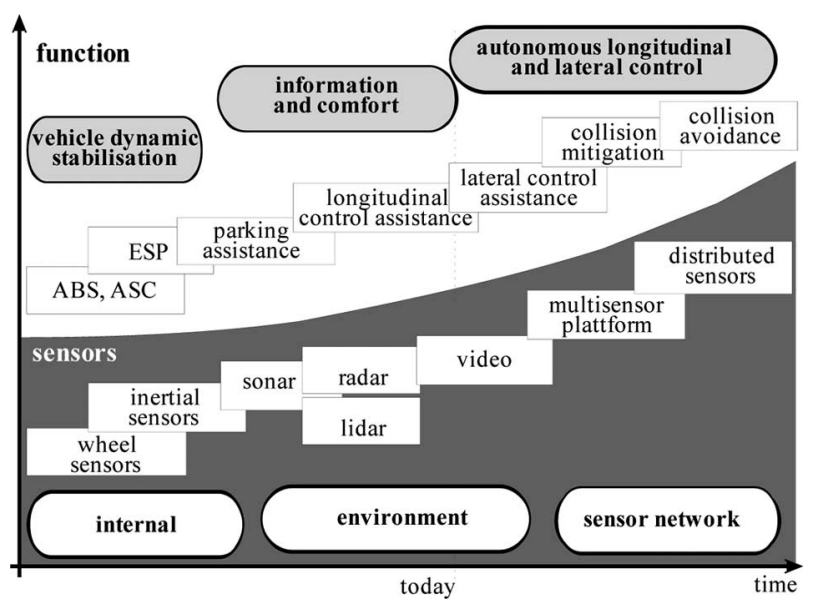

Fig. 2. Potential road map of driver assistance functions.

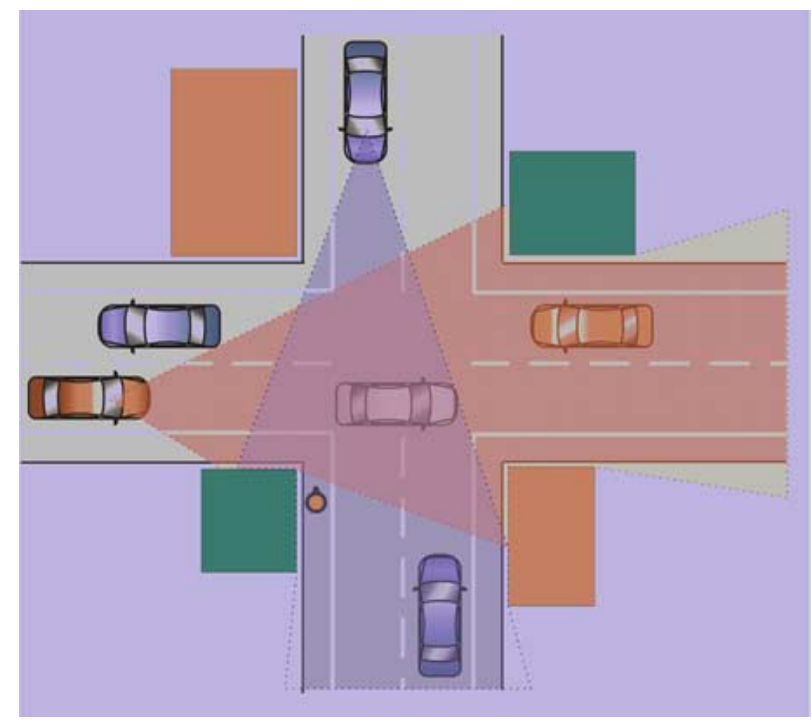

Fig. 3. Sensor network to improve perception.

perceived by the upper vehicle may not only enhance the reliability of object perception for the leftmost vehicle in the overlapping area, but is indispensable for its object detection in areas that are outside the field of perception due to limited field of view or occlusions. In those areas, cooperative perception of multiple vehicles that communicate their information provides another attractive grade of diversity. It is worth noting from the figure that cooperative sensing does not require a 100\% equipment rate, but provides benefit even at moderate rates. Multisensor platforms or sensor networks that connect vehicles may evolve to achieve a certainty level of environment perception that allows for automatic and cooperative vehicle control to mitigate or avoid collisions in future cars [27], [29], [38].

Preliminary experiments with cooperative perception between vehicles have recently been reported in [40]. An important issue in this context is the spatiotemporal registration of data transmitted in the coordinate system of other vehicles. Since the uncertainty of the spatiotemporal alignment adds itself to the intrinsic uncertainty of the sensor information, this alignment must be conducted with high precision. It is shown that an alignment strategy that combines the coarse localization information of a GPS system with the sensor output of the video sensor itself yields good results for the envisaged application.

Clearly, improvements of machine perception by fusion of sensor information should be accompanied by enhancement of the individual sensor capabilities. Temporal tracking is shown to improve performance significantly [3], [12]. Furthermore, feature diversity may strongly contribute to the robustness of sensors. In particular, video sensors offer a wide spectrum of diverse features. Fusion of disparity and motion, shape, appearance, edges, symmetry, and other 


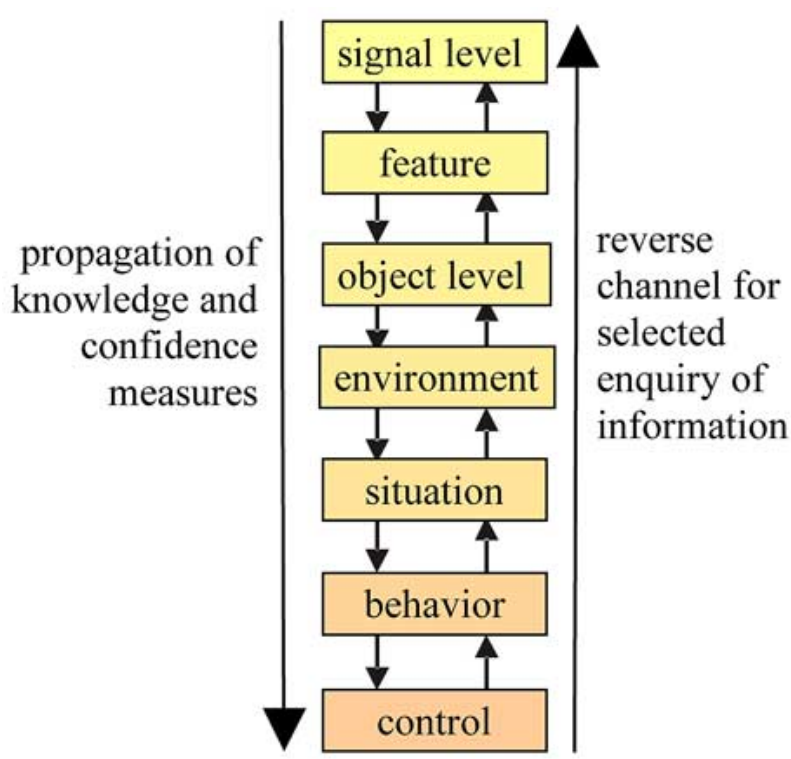

Fig. 4. Bidirectional propagation through the perception chain.

features are reported in literature, e.g., [9], [13], [15], [18], and [46]. Lidar-based simultaneous localization and mapping has been reported to yield a detailed geometric scene representation accounting for the needs of complex urban environments [44].

On the cognitive level, the achievement of situation awareness is considered an important open issue by many researchers [28]. Vehicular sensing should not be restricted to conventional metrology that acquires a couple of parameters from the scene, such as position and speed of other vehicles. Instead, a transition to consistent scene understanding is desirable. This requires a consistent propagation of knowledge and measures for its confidence throughout the perception chain, as depicted in Fig. 4. The ambitious goal is to expressively formulate at every level the ambiguity of knowledge that might stem from (sensor) noise, the signal interpretation process, or the ambiguity of previous levels. Thus, safety measures can be assigned to any potential behavior at the control level considering all previous processing. In the reverse direction, selective information can be required from previous processing units, e.g., sensor attention may be directed towards the most relevant objects in the scene. The numerous closed loops in this structure may motivate concerns and require a theory on perception stability.

\section{B. Infrastructure Development}

In the previous section, we have mentioned vehicle-tovehicle and vehicle-to-infrastructure communication as providing enhancements to the sensing capabilities of an individual vehicle. One other route for sensing enhancement is having the environment cooperate as a sensed entity.
A number of infrastructure aids have been, and are being considered for autonomous driving. Through a number of years, and most notably during Demo'97, a series of magnets were placed in the middle of a highway lane to help orient the vehicles along the lane and, to a lesser extent, provide information on upcoming curves. This was primarily advocated by PATH [47] and used by a number of other research organizations. Another technology used for the same purpose was a radar-reflective stripe placed on the lane, which the vehicle could straddle. The radar reflective stripe [35] developed by The Ohio State University was also shown in Demo'97. Through the years, other technologies have been introduced and demonstrated by different organizations.

One can mention other infrastructure-based technologies that can aid in autonomous driving, albeit indirectly. These are RFID tags that can be placed on roadways, intersections, traffic signs, bus stops, etc., or other wireless broadcast capabilities that can provide information about intersections, road clearance, vehicle approach, and location in general.

Off-road driving, of course, does not utilize any infrastructure aids. In fact, the Grand Challenge event forbade any wireless contact with the racing vehicles. However, for future applications on the roadway, it is clear that such communication would be helpful.

\section{THE DARPA GRAND CHALLENGE}

\section{A. Background}

In July 2002, the Defense Advanced Research Projects Agency (DARPA) announced a Grand Challenge for unmanned and autonomous off-road ground vehicle development. Vehicles and development teams were to be entirely self funded. The vehicles should be able to navigate a course of several hundred miles of off-road terrain in the desert southwest region of the United States, following a path defined by a series of waypoints unknown to the teams until immediately before the race, and negotiating natural and manmade obstacles and terrain features without outside intervention. Once the race began, no contact or communication with the vehicle or its systems was allowed.

DARPA conducted the first Grand Challenge event in March 2004. The course, defined by over 2000 waypoints, would take the vehicle across approximately 175 miles, beginning at Barstow, CA, and ending at Primm, NV. A prize of $\$ 1$ million (U.S.) would be awarded to the fastest vehicle that completed the course in less than ten hours. Nobody won-in fact; the furthest distance traveled was 7.2 miles.

The second Grand Challenge event was held in October 2005. A 150-mile course defined by almost 3000 waypoints began and ended at Primm, NV. This time, five teams finished the course (four within the 

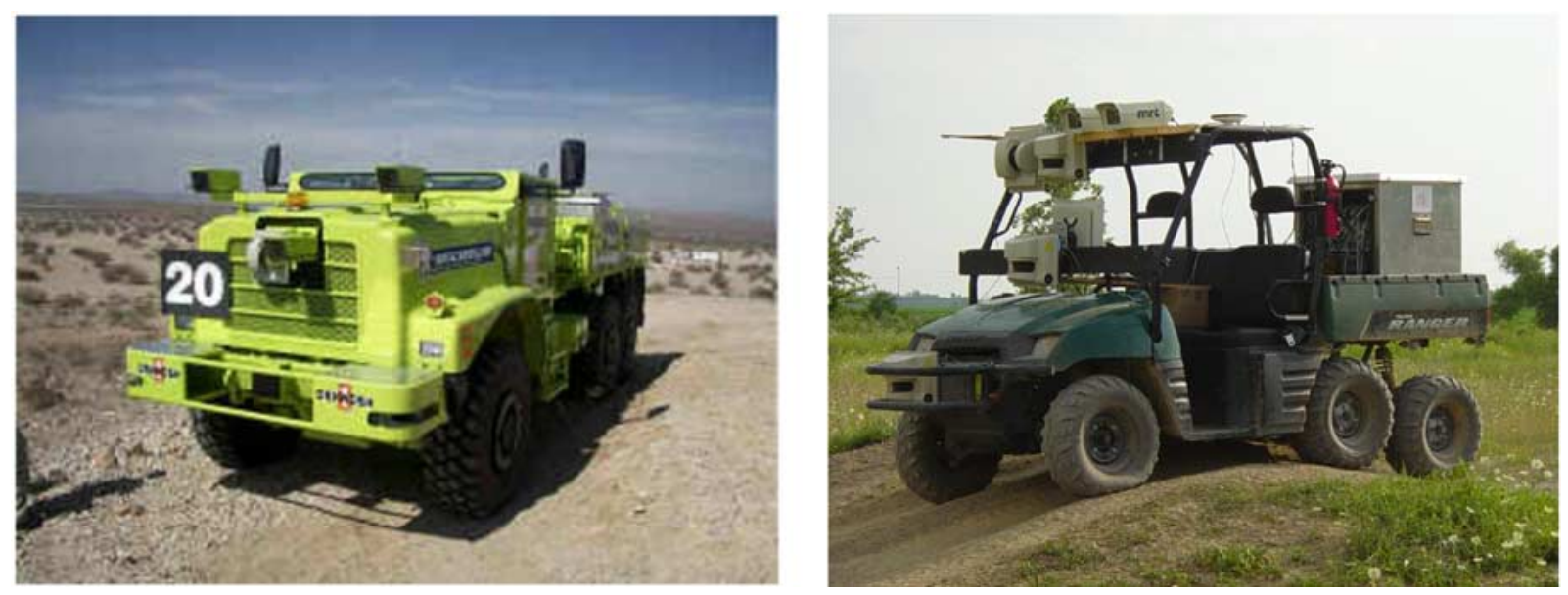

Fig. 5. TerraMax before GC'O4 and ION before GC'05.

allowed ten hours), and the vehicle Stanley, developed by the Stanford Racing Team, took the \$2 million (U.S.) prize by completing the course in 6 hours and 53 minutes, with an average speed of $19.1 \mathrm{mph}$. (http://www.darpa. mil/grandchallenge05/).

The Grand Challenge involved a number of severe challenges.

- Sensing of vehicle environment and state in a complicated, semistructured environment.

- Long-term autonomy and control over an unknown course and terrain.

- Long term robustness of both hardware and software in a bumpy, dusty, hot, and occasionally wet environment.

- Insuring safe performance of the vehicle in the absence of an on-board human driver.

- Functionality on $100 \%$ of the path.

- Significant testing and validation.

For the 2004 Grand Challenge. our team, in partnership with Oshkosh Truck Corporation, developed Terramax'04, an Oshkosh Truck Corporation Medium Tactical Vehicle Replacement (MTVR) six-wheeled heavy duty vehicle as our autonomous off-road vehicle entry. Of 106 applicants, it traveled sixth furthest (see Fig. 5).

In 2005, our "Desert Buckeyes" team outfitted ION: the Intelligent Off-Road Navigator, based on the (much smaller) $6 \times 6$ Polaris Ranger utility vehicle [6]. As in 2004, we provided drive-by-wire capability to control steering, throttle, brakes, and transmission. Various sensors, including GPS, inertial measurement units, multiple digital cameras and image processing systems, LIDARS, radars, and other sensors were integrated. Additional electrical power generation, equipment enclosure and cooling, and safety systems were also implemented. Of the 196 applicants, ION traveled 29 miles and tenth furthest.

\section{B. Off-Road Versus Highway and City Driving}

Of course, there are many differences between offroad, fully autonomous desert racing and on-road highway and urban driving, but a number of parallel challenges can also be identified.

Robustness and safe performance of hardware and software is obviously required for both off-road driving and production automobiles. Certain problems are mitigated when a system is designed for a passenger vehicle environment. Nevertheless, business concerns including marketing and liability protection demand a high level of robustness.

Sensing challenges remain, although systems can be tailored to the intended driving environment. Highway driving is a much more structured environment than offroad racing, and that structure can be exploited in sensor and control system design [33], [43]. On the other hand, the urban environment, which may consist of irregular and changing road networks and vehicles and pedestrians behaving unpredictably, is much less structured than highway driving and may require significantly more sensing capability [13], [22], [23]. Redundancy of sensing modalities is required, especially in a less structured, more uncertain environment, but the cost of many of the sensors used on Grand Challenge entries would be prohibitive for a passenger vehicle application.

The level of autonomy is also a significant design issue in a passenger vehicle system. The driver is to be part of the sensing and control loop, which means that driver attention and situation awareness, human-machine interface and driver workload considerations, and driver state considerations must be addressed.

The availability of a priori data, from the various terrain and digital elevation map and satellite imagery datasets that might be considered useful for fully autonomous offroad route planning and navigation, to the road map 
datasets, traffic condition reports, and road maintenance activity schedules that would be useful for passenger vehicle driving enhancements is also a significant issue [42]. Error correction and real-time updates, not allowed in the Grand Challenge event, are obviously useful and necessary for future vehicle systems.

Finally, as outlined in Section II-A, vehicle-to-vehicle and vehicle-to-infrastructure communication capabilities will almost certainly be involved in future vehicle systems, opening the potential for traffic cooperativity and facilitating applications from navigation and routing systems to traffic control systems to driver warning and collision avoidance systems.

\section{SENSING AND SENSOR FUSION}

\section{A. The Sensors and Sensor Fusion on ION}

The ambitious goal of the sensing and sensor fusion module for the 2005 ION was to provide $360^{\circ}$ coverage around the vehicle, while operating in an entirely unknown environment with unreliable sensors attached to a moving vehicle platform. Budget constraints required that this be accomplished without significantly expanding the sensing hardware used in the previous year. Considering the many different kinds of targets that needed to be detected and registered, the lack of high-quality a priori information about the environment, and the presence of sensor disturbances such as dust and debris, the approach we adopted was to provide a diversified system with as many different sensors modalities as possible.

The chosen sensor suite is shown in Fig. 6. The effective range of each sensor is also indicated. Three SICK LMS-221 $180^{\circ}$ scanning laser rangefinders were mounted at three different heights: the first at $25 \mathrm{~cm}$ above the ground and scanning parallel to the vehicle body, the second at $1.0 \mathrm{~m}$ about the ground and scanning in a plane that intersected the ground approximately $30 \mathrm{~m}$ ahead of the vehicle, and the third at $2.0 \mathrm{~m}$ above the ground with the scanning plane intersecting the ground approximately $50 \mathrm{~m}$ ahead of the vehicle. The use of three lasers allowed a rough estimate of object height to be computed as the vehicle approached an obstacle. A fourth laser, not shown in the figure, is mounted at $2.0 \mathrm{~m}$ above the ground and scans in a vertical plane. This laser allows the estimate of the ground profile directly ahead of the vehicle, which is crucial in order to eliminate ground clutter and the effects of vehicle pitching and bouncing motions.

An Eaton-Vorad EV300 automotive radar with a $12^{\circ}$ scanning azimuth and 80-100 m range was also mounted parallel to the vehicle body. A stereo pair of monochrome Firewire cameras and an image processing system, described below, was also installed on the vehicle. It was rigidly mounted in solid housings and included devices for system ventilation and windscreen cleaning. Finally, eight Massa M-5000/95 ultrasonic rangefinders were mounted

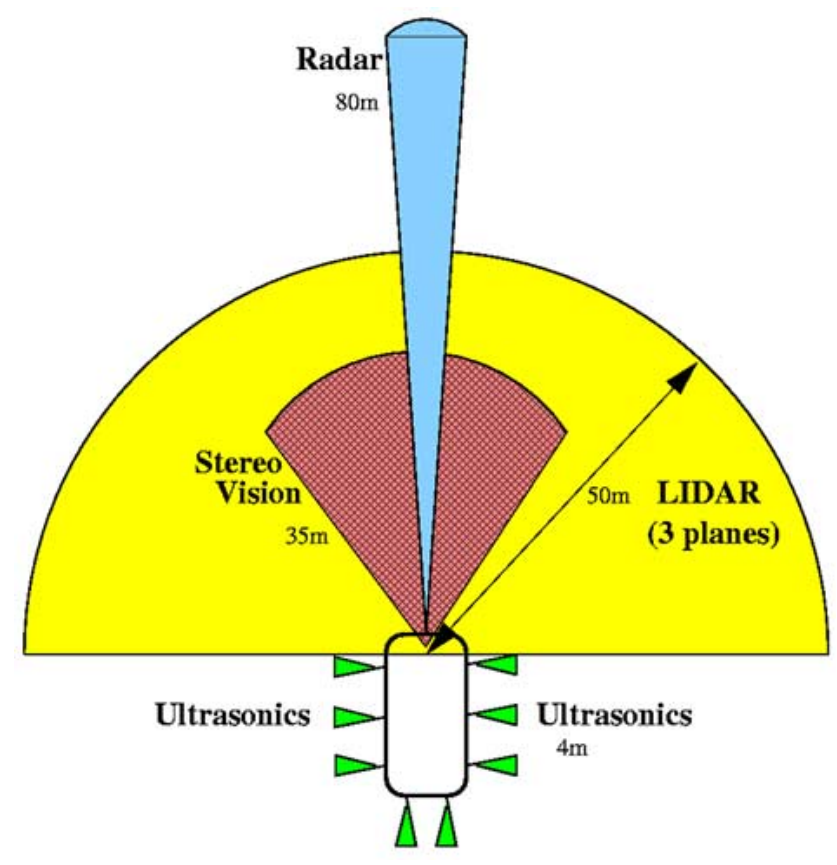

Fig. 6. Sensing system components and effective ranges.

around the vehicle to provide side sensing for narrow passages (including tunnels and bridges) and rear sensing for the vehicle, while driving in reverse. Two additional ultrasonic rangefinders were mounted high on the vehicle and angled downward at approximately $45^{\circ}$ to detect drop offs and cliff faces near the vehicle.

In addition to the sensors that monitor the external environment, localization, and orientation sensors, including a Novatel ProPak-LB-L1/L2 GPS using the Omnistar HP wide-area differential correction service, a Crossbow VG700 fiber-optic-based vertical gyroscope, a Honeywell digital compass, and wheel speed sensors on the front and back wheels were installed, validated in realtime, and fused using an extended Kalman filter to provide position, angular orientation, and speed information to both the sensor fusion and vehicle control modules.

A block diagram of the sensor fusion model is shown in Fig. 7. After sensor functionality and data integrity validation, the data from each sensor must be processed to remove any suspect or erroneous information. For the laser rangefinders, this processing includes the removal of ground clutter by culling points whose estimated heights are below the estimated ground profile. The vision system, which produces a height above ground map, is similarly processed, and areas that are marked as indeterminate are also removed from the data stream. The radar used was not observed to return ground clutter. A series of carefully constructed coordinate transformations then placed the sensor data into a unified frame of reference, while helping to insure that the fusion results from multiple sensors did not smear an object. The fused LADAR data and the vision 
data were used both to detect obstacles and detect empty space. In order to properly handle empty space detection and avoid eliminating previously detected objects, the height of previously detected objects as well as the height of the scanning plane must be considered, and an efficient ray-tracing algorithm must be utilized.

The result of the sensor fusion modules was a cellbased internal map, $160 \mathrm{~m}$ on a side, composed of 640000 cells each $20 \mathrm{~cm}$ on a side. Each cell contained three classes of confidence data: cell occupied confidence, cell empty confidence, and cell unknown confidence, as well as estimated height for an object within that cell. Placing sensor data into this map consisted of updating the three confidence measures by increasing or decreasing as appropriate, and the obstacle height estimate. The final step was generating a smaller, local map for use by the vehicle control modules by evaluating the three confidence measures and the height information. This output map includes only classified status information for each cell.

Some sample results are shown in Fig. 8(a)-(c). They show the sensor map on the left, the raw sensor data on the right, and below that a visual image of the scene. Fig. 8(a) shows the vehicle approaching a gate, Fig. 8(b) shows the vehicle approaching a tunnel, and Fig. 8(c) shows the vehicle in the tunnel.

The use of similar sensor fusion approaches for autonomous vehicles in the future is anticipated. Travel on somewhat smoother highways or city roads may not require as extensive care in establishing a "virtual horizontal plane" as was needed for the off-road terrain. Yet, versions of similar occupancy maps or extensions thereof are expected. Indeed, the possibility of multiple moving "obstacles" ahead (in highway driving), or all around (in urban driving) will make this topic even more important. Both ACC and recent advances in obstacle avoidance for highway driving have considered multiple moving "targets" ahead of the car, in the same lane or neighboring lanes. Yet, highways are somewhat more structured environments where specialized approaches may be used. Further advances in the unstructured environment of general urban driving, will require approaches more similar to those tested in the Grand Challenges.

\section{B. Vision on ION}

The structure of the video sensor platform for ION is depicted in Fig. 9, see [19]. In a preprocessing step, the

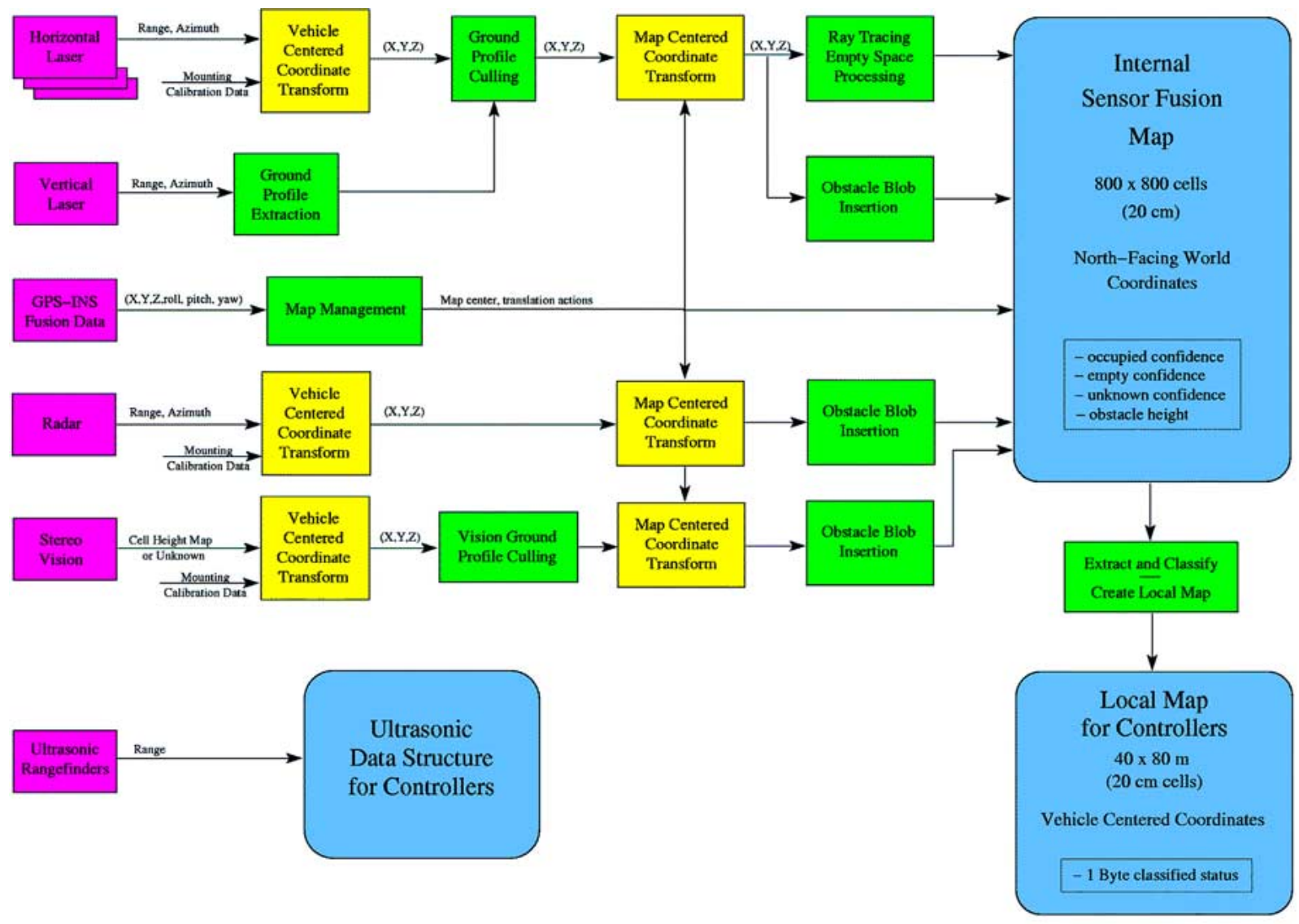

Fig. 7. Sensor fusion on ION. 


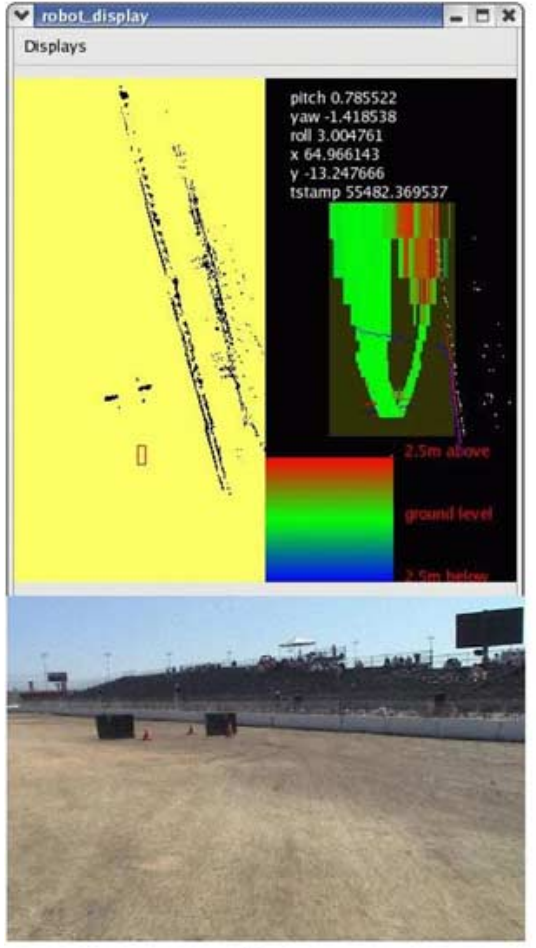

(a)

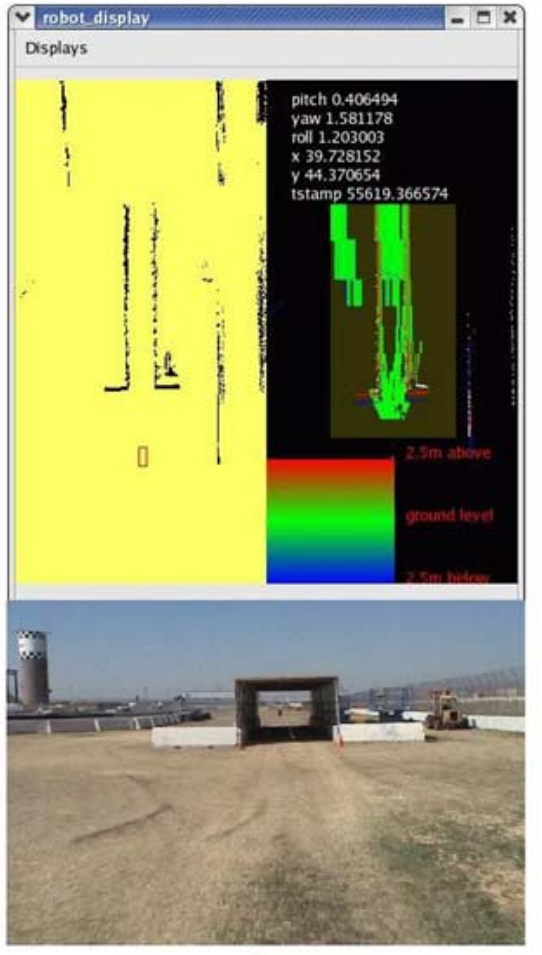

(b)

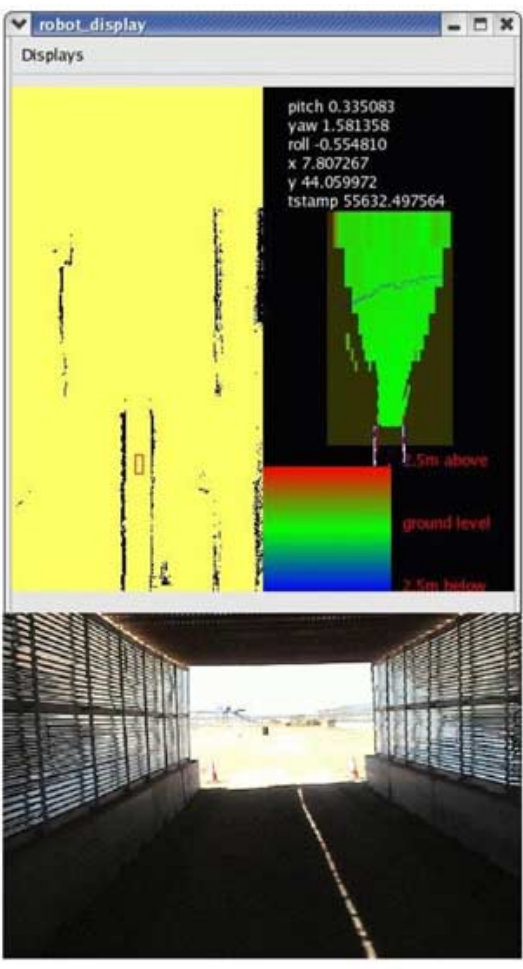

(c)

Fig. 8. (a)-(c) Three situations in the GC'05 qualifications, as seen by cameras and shown by sensor data and cell-based map.

signal of a stereoscopic camera system is compensated for lens distortion and its images are rectified, i.e., the signal is compensated for imperfect co-planarity of the two imagers.

In order to achieve a high degree of robustness against variable environmental conditions, diversity of the features is exploited by the subsequent processing step. The following three features are computed.

1) Disparity.

2) Color homogeneity.

3) Orientation.

The disparity feature allows a fast and robust computation of the ground plane parameters. Similar to estimation in Hough space, the $v$-disparity technique searches for a linear decreasing disparity along the columns [10].

Disparity is a reliable clue for depth in well-textured regions near the stereo camera. In contrast, it is highly unreliable in regions of homogeneous color. Humans possess the ability to interpolate over such regions. We aim to mimic this capability by segmenting a monoscopic image into nonoverlapping regions that include a homogeneous color. Hence, large deviations in color may only occur across region boundaries. In the absence of other cues, we then assume that either all or no points of each homogeneous region belong to the road.

Texture orientation is another clue that is used for road recognition. The eigenvector associated to the largest eigenvalue of the structural tensor $M$ serves as a measure for orientation of an image $g$ at position $\mathbf{x}$ [21]

$$
M(\mathbf{x})=\nabla g(\mathbf{x}) \nabla^{T} g(\mathbf{x})
$$

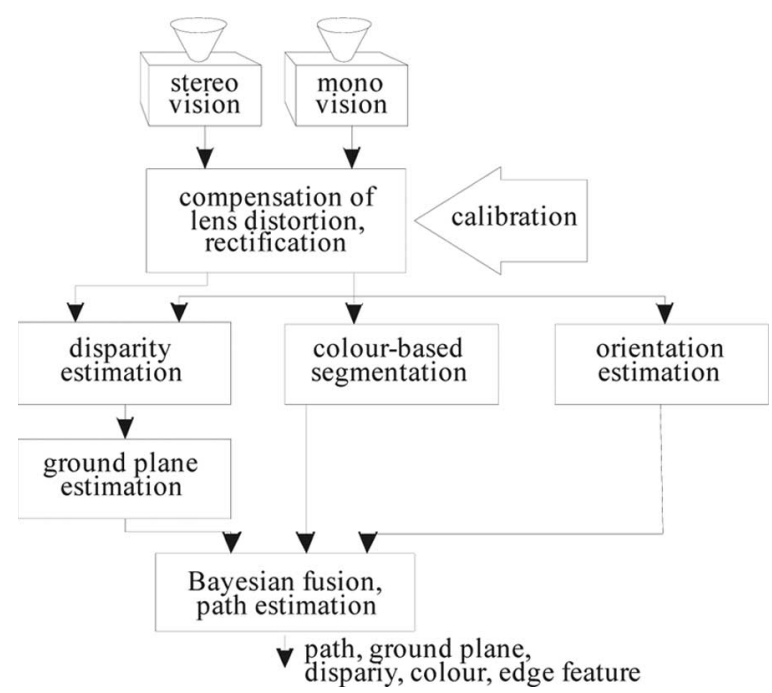

Fig. 9. ION's stereo video platform. 
The path $\mathbf{p}=\left(\mathbf{p}_{0}, \mathbf{p}_{1}, \ldots, \mathbf{p}_{N}\right)$ for given features $\mathbf{y}$ is finally estimated as the best sequence of path elements $\mathbf{p}_{i}$ in a Bayesian paradigm [14]

$$
\begin{aligned}
\hat{\mathbf{p}} & =\arg \max _{\mathbf{p}} p(\mathbf{P}=\mathbf{p} \mid \mathbf{Y}=\mathbf{y}) \\
& =\arg \max _{\mathbf{p}} \frac{p(\mathbf{Y}=\mathbf{y} \mid \mathbf{P}=\mathbf{p}) p(\mathbf{P}=\mathbf{p})}{\sum_{\mathbf{p}} p(\mathbf{Y}=\mathbf{y} \mid \mathbf{P}=\mathbf{p}) p(\mathbf{P}=\mathbf{p})}
\end{aligned}
$$

where the a priori probability $p(\mathbf{P}=\mathbf{p})$ of a path is modeled as a Markov chain of the individual path elements that favors straight composition of the individual path elements (see Fig. 10).

The likelihood $p(\mathbf{Y}=\mathbf{y} \mid \mathbf{P}=\mathbf{p})$ expresses the probability for the observed feature sequence $\mathbf{y}=\left(y_{1}, \ldots, y_{k}\right)$ of length $K$ for a known path p. In our model, we assume local features, i.e., each feature $y_{k}$ is influenced by the presence or absence of a single path element only. Assuming conditional independency among the features one can write

$$
\begin{aligned}
p(\mathbf{Y}=\mathbf{y} \mid \mathbf{P}=\mathbf{p}) & =\prod_{k=1}^{K} p\left(Y_{k}=y_{k} \mid \mathbf{P}=\mathbf{p}\right) \\
& =\prod_{\substack{k=1 \\
\mathbf{p} \neq c_{k}}}^{K} p_{0}\left(y_{k}\right) \prod_{\substack{k=1 \\
\mathbf{p} \in C_{k}}}^{K} p_{1}\left(y_{k}\right) .
\end{aligned}
$$

In this equation, $C_{k}$ denotes the set of all paths that include the path element $\mathbf{p}_{k}$ that impacts feature $y_{k} . p_{0}$ and $p_{1}$

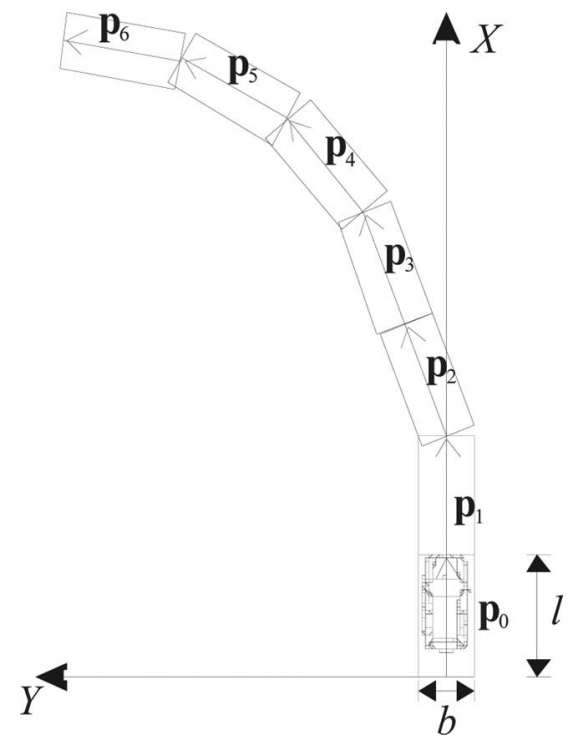

Fig. 10. Composition of the path from its elements.

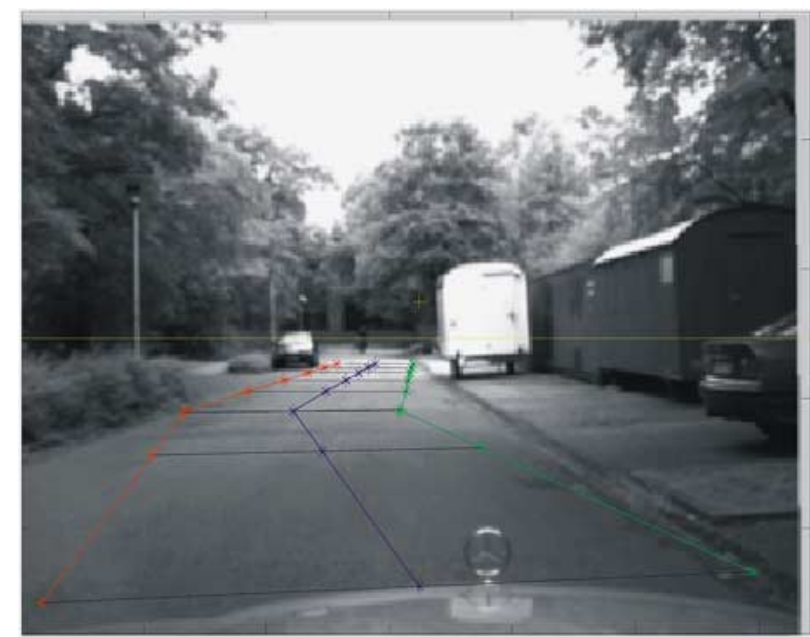

Fig. 11. Path proposed by video platform.

denote distributions of the selected features off and on the road, respectively.

The path with maximum a posteriori probability is selected in a greedy procedure using a search tree that guarantees to find a global optimum. Fig. 11 displays a left image of the stereo camera with the resulting path superimposed taken in an urban environment.

\section{CONTROL}

\section{A. High-Level Control}

"High-level control" in any autonomous driving system is based on a classification of the environment. The "situation" is understood, and a "behavior" is selected. The behavior leads to the selection of the "control" to be pursued. These levels have been shown abstractly in Fig. 4. The situation/behavior/control relationships can be modeled in terms of a finite-state machine.

Fig. 12 shows the general logic of the so-called highlevel control developed for ION and is fairly self explanatory.

Maximum speed setpoints are established a priori based on GIS map and satellite imagery information, limits imposed by the race organizers, and self-imposed limits based on our understanding of the vehicle's behavior in specific conditions. Speed setpoints are modified (reduced) in realtime based on situational awareness, including perceived obstacles, identified special circumstances, for example, a tunnel or other very narrow situation, and the current quality of the vehicle position estimates.

\section{B. Path Planning}

The DARPA race route was defined by a series of waypoints provided in the Road Data Definition File (RDDF) file format, which specified a central line and a 


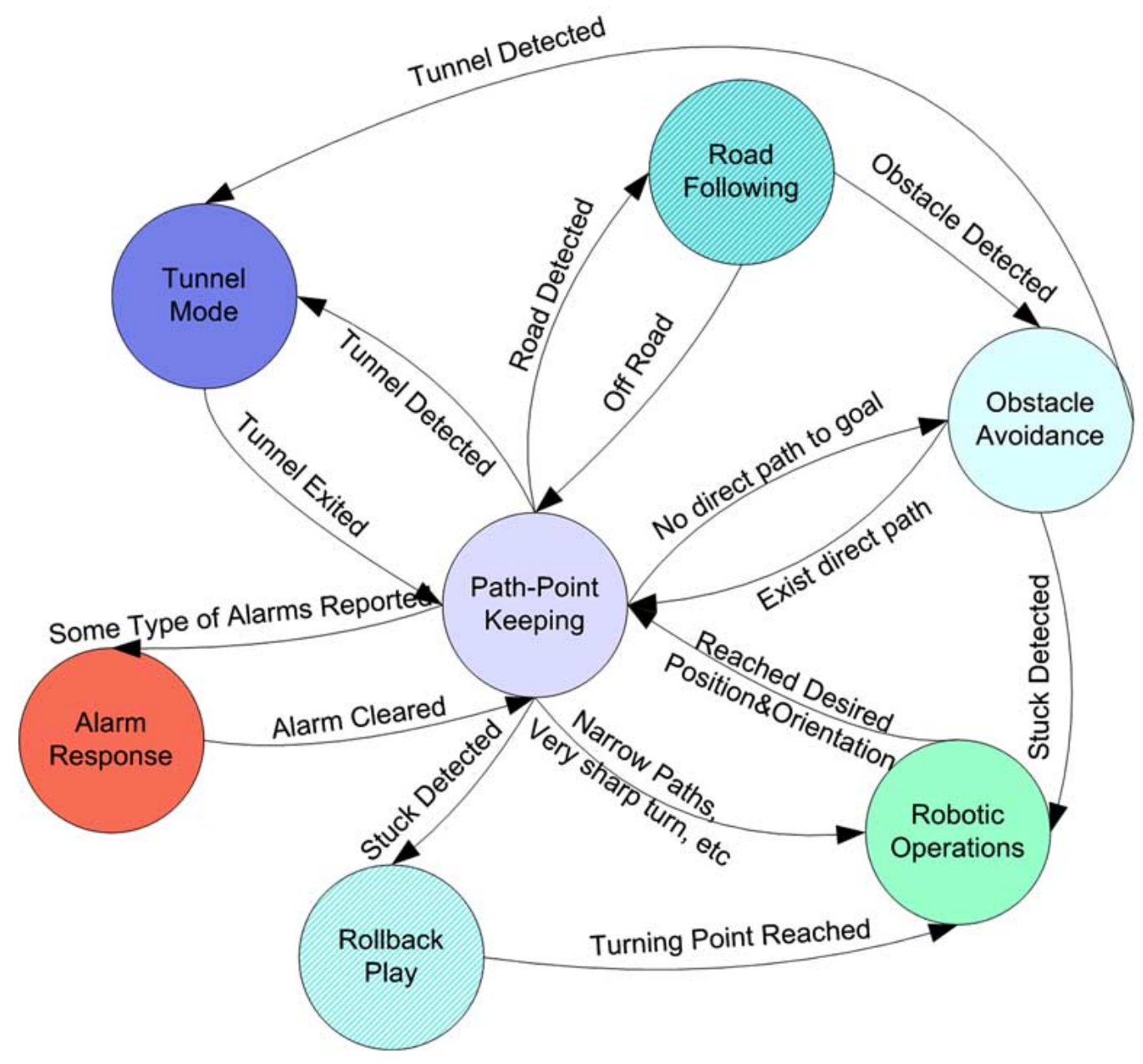

Fig. 12. Situation/behavior/control for ION.

lateral boundary width for each segment confining the vehicle trajectory to a corridor between waypoints, see Fig. 13. There were 2586 and 2935 points in the DGC 2004 and 2005 waypoint files, respectively.

Mobile mapping systems (MMSs) developed a decade ago and now widely used [5] seem to provide adequate mapping technology for the job. However, existing systems are not set up to operate in real-time.

The mapping support for the OSU DGC team included two major components: 1) providing geospatial data for automatic path planning prior to the race and 2) tools for interactive path planning in the two hours prior to the race. Automatic path planning, if needed, could have been based on the $\mathrm{A}^{*}$ algorithm, which is a generic graph search algorithm based on optimizing some cost function such as distance traveled, slope grade, negative bias for roads, higher cost for vegetated areas, and so on. The implementation and experiences of using the $\mathrm{A}^{*}$ algorithm is not discussed here, for details see, for example, [36].

The mapping effort to provide data for path planning included identifying publicly available data, assessing the costs and benefits of committing dedicated surveys, and collecting/organizing data in a format that would effectively support the path planning algorithm. Foe example, Digital Elevation Map (DEM) data at $10-\mathrm{m}$ resolution are widely available from the United States Geological Surveys (USGS) National Elevation Dataset (NED) for most of the continental U.S. Unfortunately, the DGC area is one of the regions where only $30-\mathrm{m}$ resolution DEM exists, which provides for only a very coarse terrain description, not meeting the resolution and accuracy requirements of autonomous vehicle navigation. In overcoming this issue, for example, the Red Team from Carnegie Mellon University [8] created a massive geospatial database covering about 50000 sq. miles. However, since the waypoints provided were quite dense, such exercises turned out to be unnecessary. The only need was for lowlevel path planning.

As implemented on ION during the race, the "path planning" module in the high-level controller provides the low-level controller a local path (section) to follow, which consists of a series of GPS coordinates, called path points. 


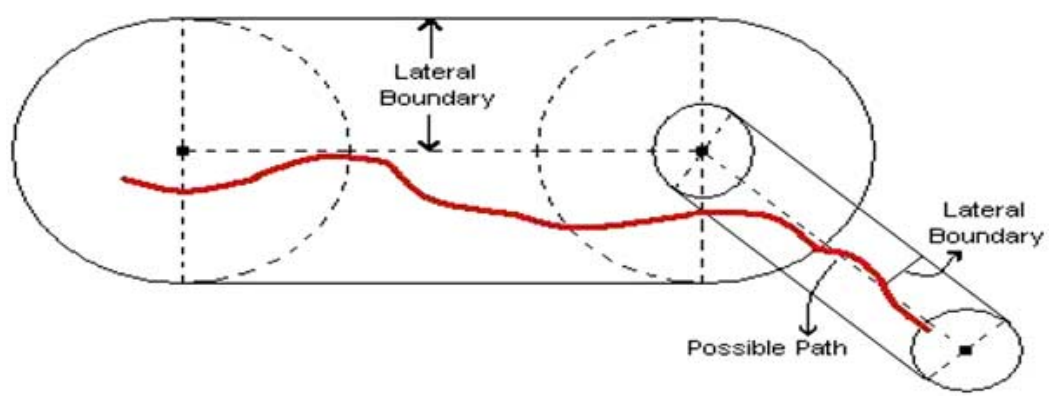

Fig. 13. DARPA waypoint corridor definition.

ION maintained ten points in each section. If the DARPA provided waypoints are too far from each other, then the "path planning" module should densify the waypoints to provide the low-level controller with an adequate waypoint distribution. The "path planning" module checks the status of the existing local path at $10 \mathrm{~Hz}$. If the path encounters some obstacles, or ION has reached the fifth point of the local path, or the "section planner" indicates that ION is getting into a new section, the "path planner" module then generates a new local path in two steps.

A smooth path, whose curvature is continuous, is generated to connect the waypoints. Four points (P0, P1, P2, P3) are provided by the "section planning" module. ION stays in the section connecting (P1, P2), P0 is the waypoint before $\mathrm{P} 1$ and $\mathrm{P} 3$ is the waypoint after P2. A cubic spline is used to generate the path. The path planning module selects the path points on the part of $\mathrm{P}(\mathrm{t})$ between the position of ION and P2. (The distance between the path points is constant, say $3 \mathrm{~m}$ ). For details, see [7].

In localities where autonomous vehicles are expected to run on roadways, much finer resolution map data will be available. Thus, the Grand Challenge terrain was not representative in either resolution or in not being constrained to road topology. On the other hand, the smooth trajectory generation issues for short distances, probably do have relevance in urban driving situations.

\section{Stopping and Swerving}

A number of issues related to constraints in sensing and control have been addressed through the years. One report that summarizes such constraints is [11].

Assuring that a vehicle has the ability to come to a complete stop in reaction to a given obstacle is one of the first criteria required to assure safe performance. The actual equation used to determine the required look ahead distance can be obtained from simple physics, and assuming no braking (Fig. 14), is given as

$$
D=\frac{v^{2}}{2(u \cos \Theta-\sin \Theta) G c}+t_{\text {react }} \times v+B
$$

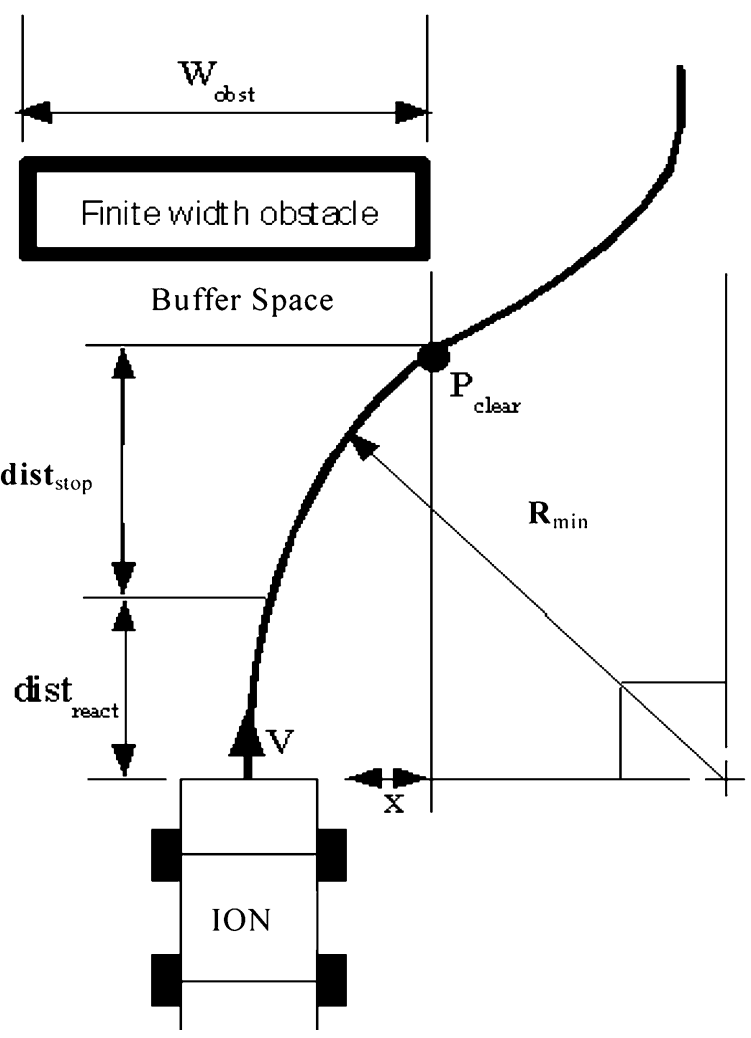

Fig. 14. Swerving around an obstacle.

The value $D$, is the look-ahead or stop distance required to assure that the vehicle can come to a complete stop before reaching the obstacle ahead. The variables in this equation are

$\begin{array}{ll}v & \text { velocity of the vehicle; } \\ u & \text { coefficient of friction; } \\ G_{C} & \text { gravity; } \\ \Theta & \text { terrain slope angle; } \\ t_{\text {React }} & \begin{array}{l}\text { time required for the vehicle to react to an } \\ \text { obstacle in sensor range; }\end{array} \\ B & \begin{array}{l}\text { addition buffer space included in calculations. }\end{array}\end{array}$


A second criterion to check is the capability to swerve around an obstacle. These calculations can help indicate items such as the maximum safe turn rate and required look-ahead distance to assure avoidance without rollover.

The equations can again be generated using simple physics (see [11] for similar developments)

$$
D=\sqrt{R^{2}-(R-X)^{2}}+V \times t_{\text {react }}+B
$$

Where $\mathrm{R}=$ maximum value of Rmin, Rroll, or Rslide

$$
\begin{aligned}
R_{\text {Roll }} & =\frac{V^{2} \times 2 h}{G c \times w} \\
R_{\text {slide }} & =\frac{V^{2}}{G c \times u} .
\end{aligned}
$$

These equations involve many critical variables because of the large number of environmental and vehicle factors that effect the swerving abilities of a vehicle. The variables used in these equations are:

$h \quad$ the height of the center of gravity;

$t_{\text {React }}$ time required for the vehicle to react to an obstacle in sensor range;

$B \quad$ Addition buffer space included in calculations; W $\quad 1 / 2$ width of obstacle;

$R$ min minimum turning radius of vehicle.
If a vehicle has braking capability, stopping distance calculations are obviously different.

A related issue for cars on roadways would be in changing lanes. For details of smooth lane-change, see [16].

One other safety related constraint that would limit the speed is consideration of rollover. Indeed, this constraint did affect us in speed selection during the GC'05.

\section{Obstacle Avoidance and Road Following}

One of the key problems in off-road driving is obstacle avoidance beyond simply swerving. Indeed, the major activity that our vehicle ION was involved in was in shortdistance path planning, or said another way, real-time path adjustment to avoid the multiple obstacles on its way (Fig. 15). A somewhat generic chart outlining this operation is given in Fig. 16. Such an activity assumes that a sensor module indicating possible feasible directions, or an occupancy map is available to form the basis for the selection of motion directions. Although this problem has been addressed by many researchers in robotic path planning, the off-road domain provides many three-dimensional problems and the race environment imposes serious time constraints.

It could be claimed that off-road driving would not require lane following capability. However, the routes in both the GC'04 and GC'05 were set up such that a major portion, indeed almost all, of the race was held on dirt roads. This fact implied that lane detection capability for

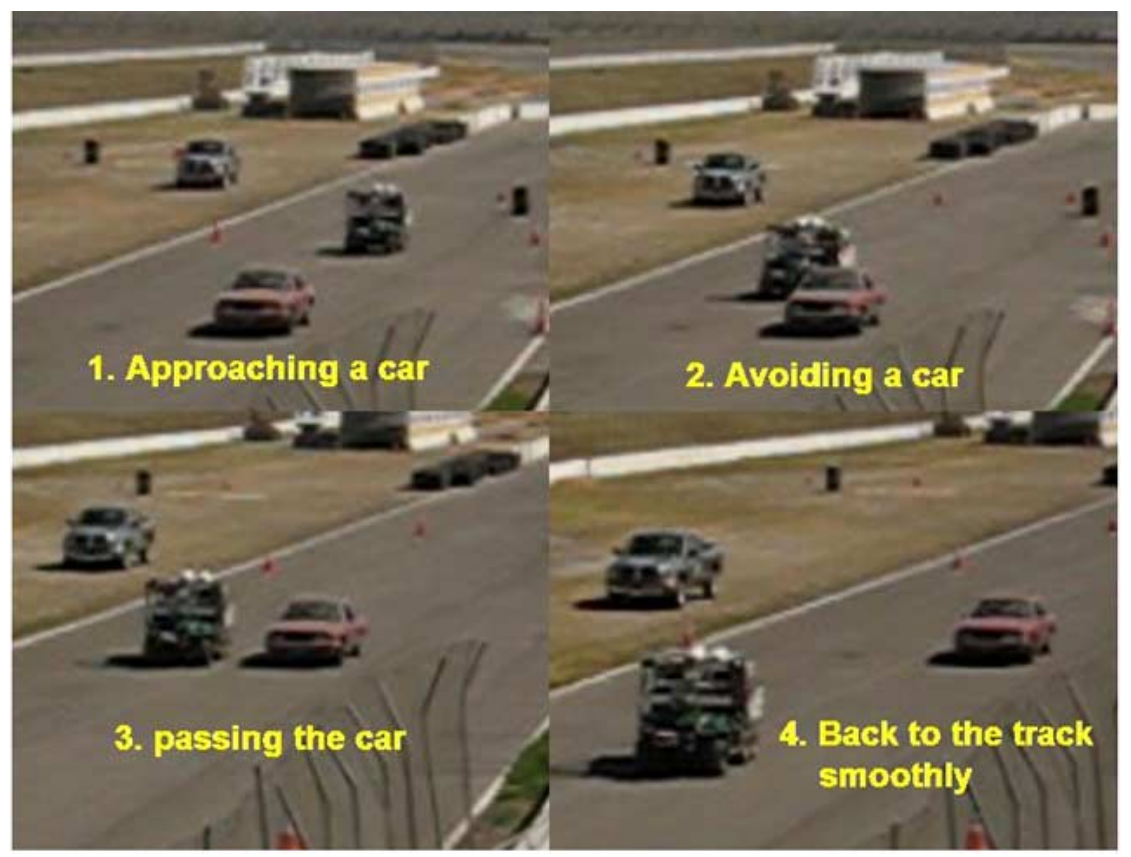

Fig. 15. ION swerving around "obstacles" at the GC'05 qualifier. 


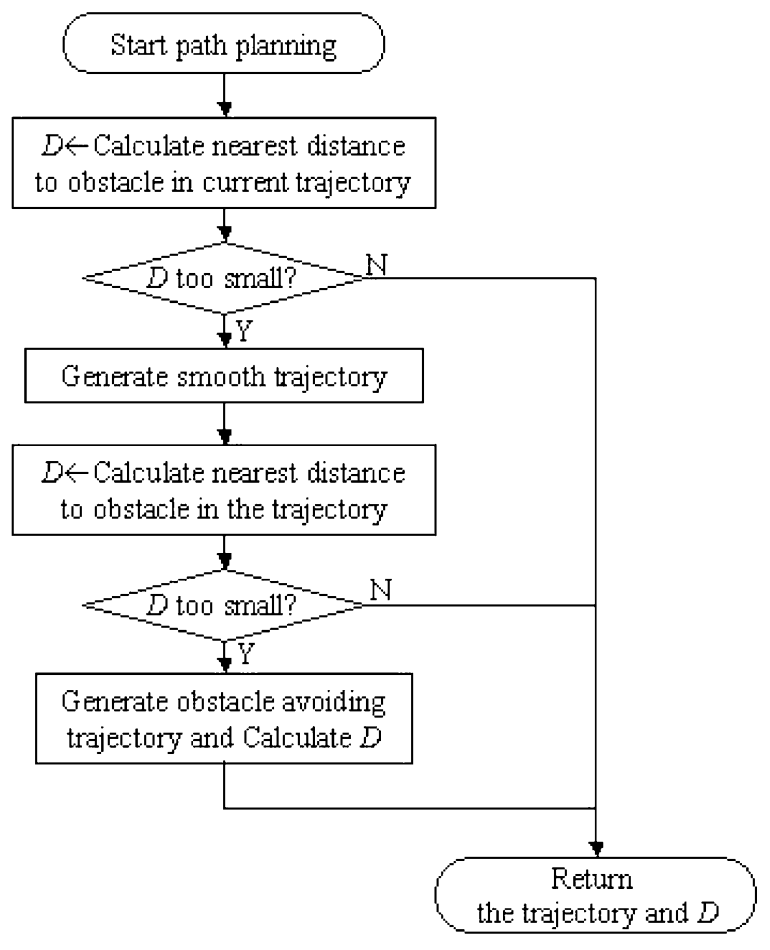

Fig. 16. Generic real-time path adjustment.

a dirt road would be useful and indeed could be used in much the same way that lane following is being advocated for Automated Highway Systems (AHS). As such, the lane following control approach used would be similar to those utilized with vision (as described in Section II-B.) or radar-reflective stripe technologies [32], and would have a "look-ahead" point and command steering based directly or indirectly depending on road curvature or offset at the look-ahead point.

\section{CONCLUSION}

\section{A. Lessons Learned}

Despite the need to enhance sensor technology and algorithms beyond the state-of-the-art, the practical performance of today's autonomous vehicles is governed by robustness and improvements due to significant on-terrain testing of hardware and algorithms. The successful teams have mainly employed well-tested hardware and software achieved by sound engineering.

Furthermore, the successful teams in the Grand Challenge were those that had more time and experience in testing. The DARPA Grand Challenge was an experimental program, yet three of the four finishing teams had the full support of professional vehicle manufacturing operations and extensive testing capabilities. These capabilities were used both at the component and the integrated system level. It is this capability that should reassure the public of the safety of future technologies in cars.

\section{B. Contributions to Future Cars}

A number of technologies and approaches were evaluated in the DARPA Grand Challenges of 2004 and 2005.

- Identifying lanes with sensors was already on its way to maturity. Here, the newer aspect was identifying and evading obstacles, reliably and fast.

- Use of GPS and map data in real-time and reliably was also evaluated. Loss of GPS signals, and retaining driving capability was an important feature of the successful vehicles. We shall see more of this in future cars.

- Although autonomous roadway driving will probably be on fairly smooth surfaces, techniques from the Grand Challenge developed for off-road driving will come in useful in possible bumpy situations on regular roads.

- Full "surround sensing" is still fairly new. Starting from experiences in the Grand Challenge, much work needs to be done for eventual utilization in autonomous urban driving scenarios.

A key issue for autonomous vehicles is perceptual reliability. It is expected that utilization of diversity across multiple levels will contribute to environment perception at a level of reliability that allows realization of safety functions. While the cooperative exploitation of diverse features as well as of information from diverse sensors has frequently been addressed in literature, little work has been reported so far on cooperative sensing between vehicles in mixed traffic.

A change of paradigm is needed in metrology for vehicles: rather then estimating a couple of parameters (like position and speed of obstacles), scene understanding is a requirement for future functions.

Very few innovations can be expected to have as high an impact on future cars as the capability to perceive the environment and to plan and conduct appropriate driving behavior. We expect that the recently announced DARPA Urban Challenge (http://www.darpa.mil/grandchallenge) will provide abundant advances in the technologies reviewed in this paper.

\section{REFERENCES}

[1] J. S. Albus and A. M. Meystel, Engineering of Mind: An Introduction to the Science of Intelligent Systems. New York: Wiley, 2001.
[2] A. Avila, G. Korkmaz, Y. Liu, H. Teh, E. Ekici, F. Ozguner, U. Ozguner, K. Redmill, O. Takeshita, K. Tokuda, M. Hamaguchi, S. Nakabayashi, and H. Tsutsui, "A complete simulator architecture for inter-vehicle communication based intersection warning systems," in Proc. IEEE Conf. Intell. Transp. Syst., Vienna, Austria, 2005, pp. 461-466.

[3] Y. Bar-Shalom, Multitarget-MultisensorTracking: Applications and Advances. Boston, MA: Artech House, 2000. 
[4] A. Broggi, M. Bertozzi, A. Fascioli, and G. Conte, Automatic Vehicle Guidance: The Experience of the ARGO Vehicle. Singapore: World Scientific, Apr. 1999.

[5] D. Grejner-Brzezinska, "Mobile mapping technology: Ten years later, Part II," Surveying and Land Information Systems, vol. 61, no. 3, pp. 83-100, 2001.

[6] Q. Chen, U. Ozguner, and K. Redmill, "Ohio State University at the 2004 DARPA grand challenge: Developing a completely autonomous vehicle," IEEE Intell. Syst., vol. 9, no. 5, pp. 8-14, Sep. 2004

[7] Q. Chen and U. Ozguner, "Intelligent off-road navigation algorithms and strategies of team desert buckeyes in the DARPA grand challenge '05," J. Field Robotics, vol. 23, no. 9, pp. 729-744, Sep. 2006.

[8] CMU Team, "High speed navigation of unrehearsed terrain: Red team technology for grand challenge 2004," CMU Rep. CMU-RI-TR_04-37, 2004.

[9] T. Dang, C. Hoffmann, and C. Stiller, "Fusing optical flow and stereo disparity for object tracking," in Proc. IEEE Int. Conf. Intell. Transp. Syst., Singapore, Sep. 2002, pp. 112-117.

[10] T. Dang and C. Hoffmann, "Fast object hypotheses generation using 3-D position and 3-D motion," in Proc. IEEE Int. Workshop on Machine Vision for Intell. Veh., Jun. 2005, p. 56

[11] Robotic Systems Technology, "Demo III experimental unmanned vehicle (XUV) program; Autonomous mobility requirements analysis," 1998, Revision 1.

[12] E. D. Dickmanns and T. Christians, "Relative 3-D state estimation for autonomous visual guidance of road vehicles," in Proc. 2nd Conf. Intell. Autonomous Syst., 1989, pp. 683-693.

[13] U. Franke, D. Gavrila, S. Görzig, F. Lindner, F. Pätzold, and C. Wöhler, "Autonomous driving goes downtown," IEEE Intell. Syst., vol. 13, no. 6, pp. 40-48, Oct. 1998.

[14] D. Geman and B. Jedynak, "An active testing model for tracking roads in satellite images," IEEE Trans. Pattern Analy. Mach. Intell., vol. 18, no. 1, pp. 1-14, Jan. 1996.

[15] M. Haag and H. H. Nagel, "Combination of edge element and optical flow estimates for 3D-model-based vehicle tracking in traffic image sequences," Int. J. Comput. Vision, vol. 35, no. 3, pp. 295-319, 1999.

[16] C. Hatipoglu, U. Ozguner, and K. A. Redmill, "Automated lane change controller design for Intelligent Transportation Systems," IEEE Trans. Autom. Control, vol. 4, no. 1, pp. 13-22, Mar. 2003.

[17] K. Hiramatsu, K. Satoh, M. Iguchi, M. Kume, T. Onoda, and S. Mori, "Design principles of the advanced safety vehicle," in Proc. 7th World Congr. Intell. Transp. Syst., Nov. 2000, Paper Number 4242. [CD-ROM].
[18] C. Hoffmann, T. Dang, and C. Stiller, "Vehicle detection fusing 2-D visual features," in Proc. IEEE Intell. Vehicles Symp., Parma, Italy, Jun. 2004, pp. 280-285.

[19] B. Hummel, S. Kammel, T. Dang, C. Duchow, and C. Stiller, "Vision-based path-planning in unstructured environments," in Proc. IEEE Intell. Veh. Symp., Tokyo, Japan, Jun. 2006, pp. 176-181.

[20] S. Ishida and J. E. Gayko, "Development, evaluation and introduction of a lane keeping assistance system," in Proc. IEEE Intell. Veh. Symp., Jun. 2004, pp. 943-944.

[21] B. Jähne, Practical Handbook on Image Processing for Scientific Applications. Boca Raton, FL: CRC Press, 1997.

[22] T. M. Jochem, D. A. Pomerleau, and C. E. Thorpe, "Vision-based neural network road and intersection detection and traversal," in Proc. IEEE Conf. IROS, Aug. 1995, pp. 344-349.

[23] H. Jula, B. Kosmatopoulos, and P. Ioannou, "Collision avoidance analysis for lane changing and merging," IEEE Trans. Veh. Technol., vol. 49, pp. 2295-2308, 2001.

[24] K. Kodaka and J. Gayko, "Intelligent systems for active and passive safety-Collision mitigation brake system," in Proc. ATA EL Conf., Parma, Italy, 2004.

[25] L. A. Klein, Sensor and Data Fusion: A Tool for Information Assessment and Decision Making. Bellingham, WA: SPIE Press, Monograph vol. PM138, 2004.

[26] Y. Liu, U. Ozguner, and T. Acarman, "Performance evaluation of inter-vehicle communication in highway systems and in urban areas," IEE Proc. ITS, vol. 153, no. 1, pp. 63-75, Mar. 2006.

[27] M. Maurer and C. Stiller, Eds., Fahrerassistenzsysteme mit maschineller Wahrnehmung. Berlin, Germany: Springer-Verlag, 2005.

[28] H. H. Nagel, "Steps toward a cognitive vision system," AI-Magazine, vol. 25, no. 2, pp. 31-50, 2004.

[29] E. Nett, M. Gergeleit, and M. Mock, "Mechanisms for reliable cooperation of vehicle," in Proc. 6th Int. Symp. High Assurance Syst. Eng., Oct. 2001, pp. 75-81.

[30] G. Nöcker, D. Hermann, and A. Hiller, "Telematics based traffic organization," presented at the 7th World Congr. Intell. Transp. Syst., 2000, paper no. 2013.

[31] K. Redmill, U. Ozguner, and A. Broggi, "Team TerraMax and the DARPA grand challenge: A general overview," in Proc. Intell. Veh. Symp. 2004, pp. 232-237.

[32] K. Redmill, "A simple vision system for lane keeping," in Proc. IEEE Intell. Transp. Syst. Conf., Nov. 9-12, 1997, pp. 212-217.

[33] K. Redmill, S. Upadhya, A. Krishnamurthy, and U. Ozguner, "A lane tracking system for intelligent vehicle applications," in Proc. IEEE Intell. Transp. Syst. Conf., Aug. 25-29, 2001, pp. 273-279.
[34] K. A. Redmill and U. Ozguner, "The Ohio State University automated highway system demonstration vehicle," presented at the 1998 SAE World Congr., SAE Tech. Pap. \\#980855.

[35] K. Redmill and U. Ozguner, "The OSU demo '97 vehicle," in 1997 IEEE Intell. Transp. Syst. Conf., Oct. 9-12, 1997, pp. 502-507.

[36] S. Skiena, Dijkstra's Algorithm, in Implementing Discrete Mathematics: Combinatorics and Graph Theory With Mathematica. Reading, MA: Addison-Wesley, 1990, pp. 225-227.

[37] C. Stiller, J. Hipp, C. Rössig, and A. Ewald, "Multisensor obstacle detection and tracking," Image and Vision Comput. J., vol. 18, no. 5, pp. 389-396, Apr. 2000.

[38] C. Stiller, "Cooperative environment perception," in Proc. 1st AUTOCOM Workshop on Preventive and Active Safety Systems for Road Vehicles, Istanbul, Turkey, Sep. 2005. pp. 39-41.

[39] A. Takahashi and N. Asanuma, "Introduction of Honda ASV-2 (advanced safety vehicle-Phase 2)," in Proc. AVEC 5th Int. Symp. Advanced Veh. Control, Ann Arbor, MI, 2000, pp. 449-454.

[40] K. Tischler, M. Clauss, Y. Günter, N. Kämpchen, R. Schreier, and M. Stiegeler, "Networked environment description for advanced driver assistance systems," in Proc. IEEE Intell. Transp. Syst. Conf., Vienna, Sep. 2005, pp. 785-790.

[41] C. Thorpe, T. Jochem, and D. Pomerleau, "The 1997 automated highway free agent demonstration," in Proc. IEEE Intell. Transp. Syst. Conf., Nov. 1997, pp. 496-501.

[42] C. K. Toth, E. Paska, Q. Chen, Y. Zhu, K. Redmill, and U. Ozguner, "Mapping support for the OSU DARPA grand challenge vehicle," in Proc. IEEE ITS Conf., Toronto, ON, Canada, Sep. 2006, pp. 1580-1585.

[43] K. A. Ünyelioğlu, C. Hatipoğlu, and U. Özgüner, "Design and stability analysis of a lane following controller," IEEE Trans. Control Syst. Technol., vol. 5, pp. 127-134, 1997.

[44] C. Wang, C. Thorpe, and S. Thrun, "Online simultaneous localization and mapping with detection and tracking of moving objects: Theory and results from a ground vehicle in crowded urban areas," in Proc. IEEE Int. Conf. Robotics Autom., May 2003, vol. 1, pp. 842-849.

[45] H. Winner, "Adaptive cruise control," in Automotive Electronics Handbook, R. Jurgen, Ed. New York: McGraw-Hill, 1999, ch. 30.

[46] T. Zielke, M. Brauckmann, and W. von Seelen, "Intensity and edge-based symmetry detection applied to car-following," ECCV Lecture Notes in Computer Science, vol. 588, pp. 865-873, 1992.

[47] H.-S. Tan, R. Rajamani, and W.-B. Zhang, "Demonstration of an automated highway platoon system," in Proc. IEEE 1998 ACC, vol. 3, pp. 1823-1827. 


\section{ABOUT THE AUTHORS}

ümit Özgüner (Member, IEEE) received the Ph.D. from the University of Illinois, Chicago, in 1975.

He has held research and teaching positions at IBM T.J. Watson Research Center, the University of Toronto, and Istanbul Technical University. Since 1981, he has been with The Ohio State University, where he is presently Professor of Electrical and Computer Engineering and holds the TRC Inc. Chair on Intelligent Transportation Systems (ITSs). The team he coordinated participated successfully

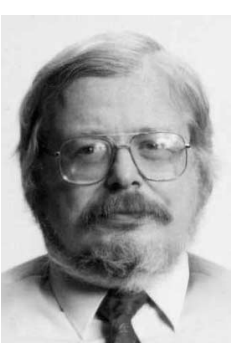
in the (US DOT supported) 1997 Automated Highway System Technology Demonstration in San Diego, where they demonstrated three fully automated cars doing lane-keeping, convoying, and passing using radar and vision-based guidance. Recently, he has been leading a group developing autonomous off-road vehicles and participated in the DARPA 2004 and 2005 Grand Challenge. He is the author of over 300 publications which have appeared in journals, as book chapters and in conference proceedings. He has been an invited plenary speaker on ITS topics in many international meetings in the U.S., Japan, China, India, Turkey, and Italy. His areas of research interest are in ITSs, decentralization, and autonomy issues in large systems.

Dr. Özgüner was the first President of the IEEE ITS Council in 1999 and reelected in 2000. He has also served the IEEE Control Society in many positions, and was an elected member of its Board of Governors (19992001). He participated in the organization of many conferences (including the IFAC Congress) and has been the General Chair of the 2002 CDC. He was the Program Chair for the 1997 IEEE ITS Conference, which he helped initiate, and served as the General Chair for the 2003 Intelligent Vehicles Symposium.

Christoph Stiller (Senior Member, IEEE) received the Diplom degree in electrical engineering (M.Sc.E.E.) and the Dr.-Ing. degree (Ph.D.) with distinction from the Aachen University of Technology, Aachen, Germany, in 1988 and 1994, respectively, where he was a Scientific Assistant. He conducted a Postdoctoral year at INRS-Telecommunications, Montreal, QC, Canada.

From 1987 to 1988, he visited the Norwegian University of Technology, Trondheim, Norway. In 1995, he joined the Corporate Research and Advanced Development of Robert Bosch GmbH, Hildesheim, Germany, where he was responsible for "Computer Vision for Automotive Applications." Since 2001, he has been a Full Professor and Head of the Institute for Measurement and Control Engineering, Karlsruhe University, Germany. He is speaker of the Karlsruhe-Munich Transregional Collaborative Research Center "Cognitive Automobiles." His present interest covers sensor signal analysis, visual inspection, video sensing, information fusion, and realtime applications thereof.

Dr. Stiller is Vice President of Member Activities of the IEEE Intelligent Transportation Systems Society. He served two terms as Associate Editor for the IEEE TRANSACtions on IMAge PRocessing from 1999 to 2003 and since 2004 serves as Associate Editor for the IEEE TRANSACTIONS ON INTELLIGENT TRANSPORTATION SYSTEMS. He serves as Chapter Chair for Measurement in Production in the German Electrical Engineering Association (VDE).
Keith Redmill (Member, IEEE) received the B.S.E.E. and B.A. Mathematics degrees from Duke University, Durham NC, in 1989, and the M.S. and Ph.D. degrees from The Ohio State University, Columbus, in 1991 and 1998, respectively.

He has been a Research Scientist in the Department of Electrical and Computer Engineering, The Ohio State University, since 1998. He is actively involved in research involving autonomous ground and aerial vehicles, and traffic and trans-

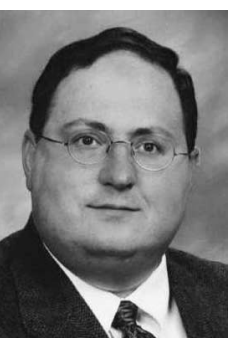
portation systems. Recently, he led the integration of autonomous offroad vehicles for the 2004 and 2005 DARPA Grand Challenges. His areas of research interest include control and systems theory, intelligent transportation systems, autonomous vehicle and robotic systems, realtime embedded systems, GPS and inertial positioning and navigation, transit and traffic monitoring, image processing, wireless digital communication for vehicles, sensor technologies, decentralized multiagent hierarchical and hybrid systems, and numerical analysis and scientific computing.

Dr. Redmill is a member of the the Society for Industrial and Applied Mathematics (SIAM). 